\title{
Study of the gamma-ray state changes of PSR J2021+4026 with Fermi-LAT
}

\section{A. Fiori, ${ }^{a, *}$ M. Razzano, ${ }^{a}$ P. M. Saz Parkinson, ${ }^{b, c}$ and R. Mignani ${ }^{d, e}$ on behalf of the Fermi-LAT collaboration}

${ }^{a}$ Dipartimento di Fisica "Enrico Fermi", Università di Pisa and Istituto Nazionale di Fisica Nucleare, Sezione di Pisa, I-56127, Pisa, Italy

${ }^{b}$ Santa Cruz Institute for Particle Physics, Department of Physics and Department of Astronomy and Astrophysics, University of California at Santa Cruz, Santa Cruz, CA 95064, USA

${ }^{c}$ Department of Physics, The University of Hong Kong, Pokfulam Road, Hong Kong, China

${ }^{d}$ INAF - Istituto di Astrofisica Spaziale e Fisica Cosmica Milano, I-20133, Milano, Italy

${ }^{e}$ Janusz Gil Institute of Astronomy, University of Zielona Gora, 65-265, Zielona Gora, Poland

E-mail: alessio.fiori@pi.infn.it

The peculiar radio-quiet PSR J2021+4026, in the Gamma Cygni supernova remnant, is one of the brightest of the over 250 pulsars detected by Fermi-LAT. It is unique in being the only known isolated gamma-ray pulsar to undergo abrupt flux changes, which are also simultaneous with spin-down variations. The first change was observed by Fermi-LAT in October 2011, and it was followed by a recovery over a timescale of 100 days around December 2014. A second change occurred in February 2018. In the last few years, PSR J2021+4026 has been widely studied at different wavelengths. We report our latest results on this source, based on a Fermi-LAT analysis of its gamma-ray variability. In particular, we have studied the changes in the spectral and timing parameters on different timescales. Our results are essential to relate the observed events to changes in the geometry of the particle acceleration regions in the pulsar magnetosphere. Therefore, this study will allow us to enhance our knowledge of this source and its behavior.

$37^{\text {th }}$ International Cosmic Ray Conference (ICRC 2021)

July 12 th - 23rd, 2021

Online - Berlin, Germany

\footnotetext{
*Presenter
} 


\section{Introduction}

The young, radio-quiet $\gamma$-ray pulsar PSR J2021+4026 was discovered with the Fermi Large Area Telescope (LAT) [1] within the shell of the Gamma Cygni supernova remnant (G 78.2+2.1). Pulsations with $P \sim 265 \mathrm{~ms}$ were detected using blind periodicity searches [2] and the pulsar was associated with the bright EGRET source EG J2020+4017. A likely X-ray counterpart was inferred using Chandra data [3] and X-ray pulsations were later observed with XMM-Newton [4].

Among the more than $250 \gamma$-ray pulsars observed by Fermi-LAT ${ }^{1}$, PSR J2021+4026 shows a unique nature. In fact, its $\gamma$-ray flux $\left(F_{\gamma} \sim 7.9 \times 10^{-10} \mathrm{erg} \mathrm{cm}^{-2} \mathrm{~s}^{-1}\right)$ varies rapidly and simultaneously with its spin-down rate $\left(\dot{P} \sim 5.4 \times 10^{-14} \mathrm{~s} \mathrm{~s}^{-1}\right)$ at intervals of a few years, periodically switching between high-flux/low-spin-down states and low-flux/high-spin-down states. The first state change (jump) was observed in October 2011 [5], when the flux dropped by $\sim 18 \%$ and the spin-down rate increased by $\sim 5.6 \%$ in a timescale $<7$ days. After a slow recovery, occurred over $\sim 100$ days around December 2014 [6] [7], a similar jump occurred in February 2018 [8]. Previous works also studied the variations of the $\gamma$-ray spectrum and of the pulse profile.

We have another example of variability among $\gamma$-ray pulsars, which is the binary millisecond pulsar PSR J1023+0038 [9]. In 2013, this source showed an increase in the $\gamma$-ray flux concurrent with a radio disappearance [10]. However, this event was modeled as a transition between a rotation-powered state and an accretion-powered state due to the presence of a companion. On the other hand, the jumps of PSR J2021+4026 were modeled as starquake-induced glitches associated to a magnetospheric reconfiguration [6]. Therefore, PSR J2021+4026 is currently the only known gamma-ray variable pulsar.

Here we present the preliminary results of an advanced maximum likelihood analysis based on the most updated Fermi-LAT data. Our purpose is to accurately measure variations in the $\gamma$-ray flux and spectrum across the jumps. We will discuss the observations in the perspective of a change in the geometry of the pulsar magnetosphere.

\section{Methods}

Data were prepared and analyzed using Fermitools $^{2}$, the standard analysis suite released by the Fermi-LAT collaboration. Our dataset covers 12 years from August 5, 2008 to May 26, 2020, and it includes all LAT photons of P8R3_SOURCE_V2 event class. We selected events in a region with a radius of $10^{\circ}$ around PSR $\mathrm{J} 2021+4026$, with zenith angles $z<90^{\circ}$ and in the energy range from $100 \mathrm{MeV}$ to $300 \mathrm{GeV}$. We binned data with 35 logarithmically spaced energy bins (10 bins per decade) and squared angular pixels of size $0^{\circ} .1$. Figure 1 shows the produced counts map.

LAT photons are partitioned into four event types (PSF0, PSF1, PSF2, PSF3) based on the quality of the angular reconstruction. Each event type has a different LAT response; therefore, we prepared four sets of binned data and performed a maximum likelihood analysis with summed PSF components. In order to study the spectral variations between different states, we further divided data in four distinct time intervals, labelled with capital Roman letters. These intervals are defined

\footnotetext{
${ }^{1}$ https://confluence.slac.stanford.edu/display/GLAMCOG/Public+List+of+LAT-Detected+ Gamma-Ray+Pulsars

2https://fermi.gsfc.nasa.gov/ssc/data/analysis/documentation/
} 


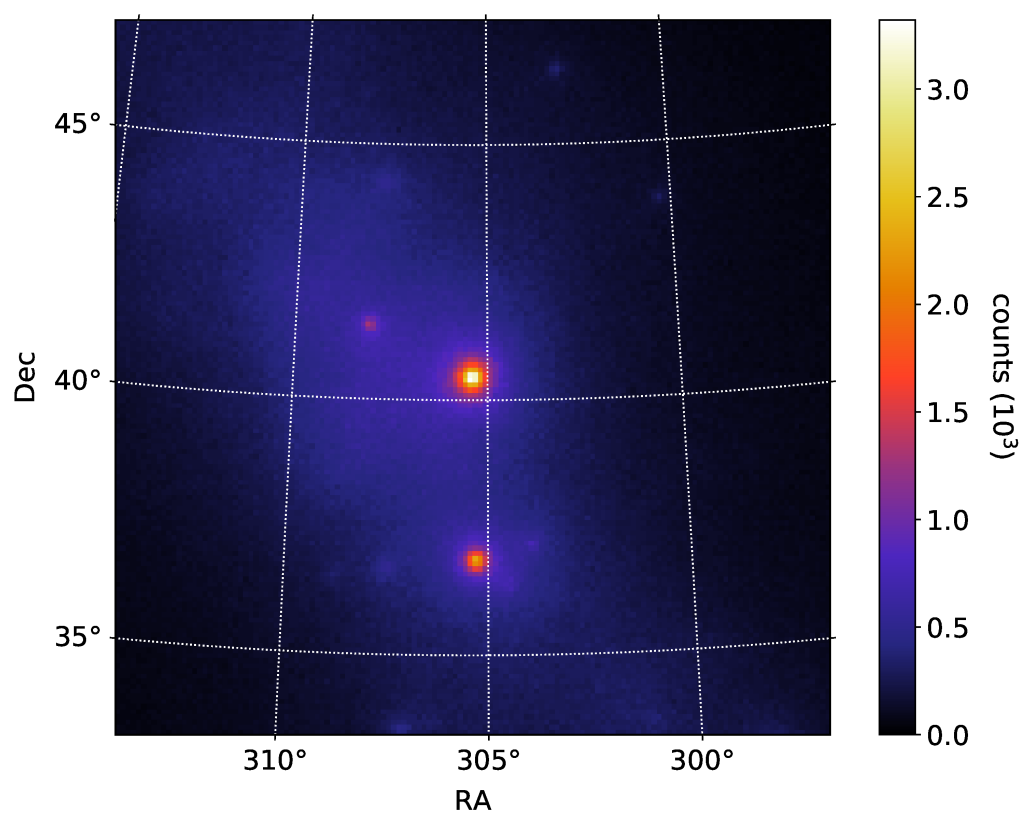

Figure 1: Binned counts map including all photons collected between August 5, 2008 and May 26, 2020. The size of the RoI is $14^{\circ} \times 14^{\circ}$. The size of the pixels is $0^{\circ} .1$. The three bright sources are PSR J2021+4026 (center), PSR J2021+3651 ( $3^{\circ} .6$ south) and PSR J2032+4027 ( $2^{\circ} .3$ north-east). The Galactic plane is visible in the background.

as follows. A: August 5, 2008 (MJD 54683) - October 16, 2011 (MJD 55850). B: October 16, 2011 (MJD 55850) - December 9, 2014 (57000). C: December 9, 2014 (MJD 57000) - February 2, 2018 (MJD 58150). D: February 2, 2018 (MJD 58150) - May 26, 2020 (MJD 58995).

We built a model of the $\gamma$-ray sky starting from the 4FGL catalog [11] and including all sources within $20^{\circ}$ from PSR J2021+4026. We also included templates for the Cygnus Loop and for the Galactic and isotropic diffuse emissions. The $\gamma$-ray spectrum of PSR J2021+4026 was modeled as a power law with an exponential cutoff,

$$
d N / d E \propto E^{-\Gamma_{1}} \exp \left(-b E^{2 / 3}\right)
$$

where the normalization and the spectral parameters were kept free. We freed the normalization of other bright pulsars (PSR J2021+3651, PSR J2032+4027) and extended sources (SNR G $78.2+2.1$, Cygnus Cocoon). No other pulsar in the field show variability; however, there are extragalactic sources with reported flaring behavior. In order to take account of spurious flux changes, we freed the normalization of all variable point sources within $7^{\circ}$. Finally, we freed the Galactic diffuse emission and fixed the isotropic diffuse emission. We ended up with 23 free parameters.

In the binned likelihood analysis, the accuracy on the results at energies below $1 \mathrm{GeV}$ was improved by applying corrections due to the energy dispersion. Moreover, we took account of systematic errors on the diffuse background by including likelihood weights, which were calculated for each event type. 


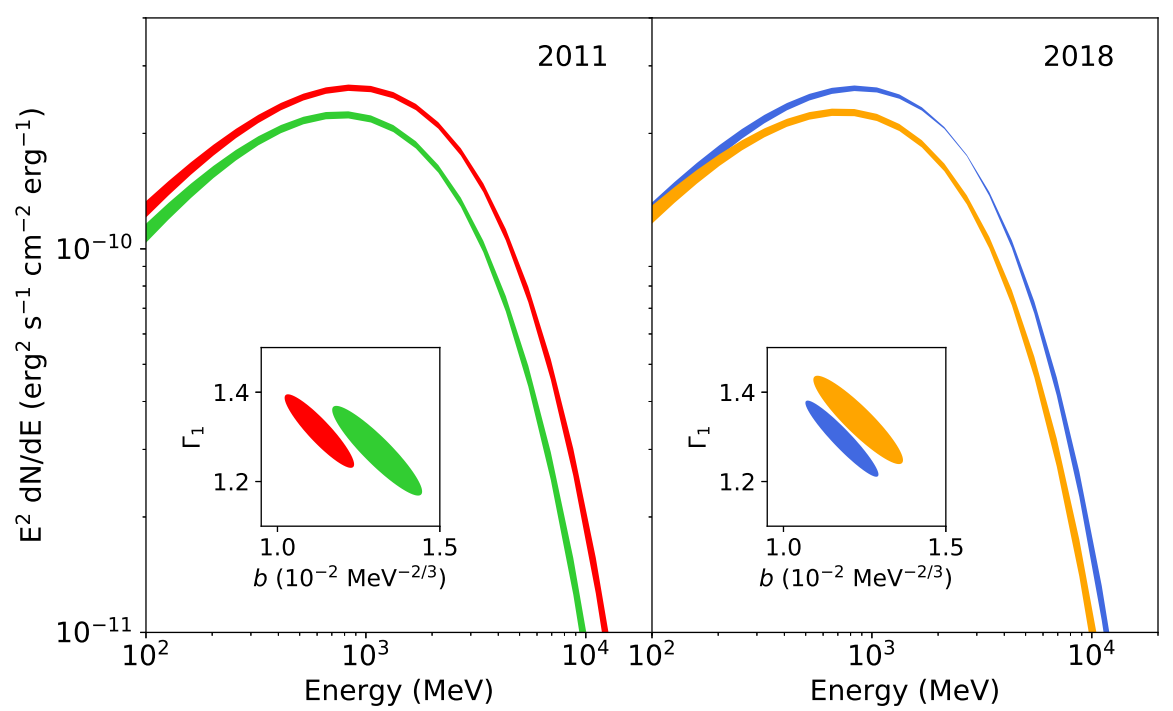

Figure 2: Fitted spectral energy distributions of PSR J2021+4026 in intervals A (red), B (green), C (blue) and D (orange) at the 2011 and 2018 jumps. The bands represent the $3 \sigma$ credibility intervals from a multivariate Gaussian distribution. The inset panels show the $3 \sigma$ credibility ellipses around the optimal values of power-law index, $\Gamma_{1}$, and exponential factor, $b$.

\section{Results}

The fits to the time intervals $\mathrm{A}, \mathrm{B}, \mathrm{C}$, and $\mathrm{D}$ show consistent relative variations in the $\gamma$-ray flux at both state changes: $\Delta F_{\gamma} / F_{\gamma}=-15.3 \pm 1.4 \%$ at the 2011 jump, $\Delta F_{\gamma} / F_{\gamma}=-16.3 \pm 1.4 \%$ at the 2018 jump. We can also observe differences in the spectrum, mainly due to variations in the exponential factor, $b$, rather that the power-law index, $\Gamma_{1}$, which does not change significantly. We also ran a fit using a model with fixed spectral parameters ( lobal). The significance of the variable model with respect to the global model is $>3 \sigma$ in $\mathrm{A}, \mathrm{B}$ and $\mathrm{D}$, while significance is lower $(2 \sigma)$ in $\mathrm{C}$. However, the pre-jump and post-jump spectra are significantly different $(3 \sigma)$ at both jumps (Figure 2).

We analyzed 30-day and 7-day time intervals in order to study short-timescale variations. Due to the reduced exposure, we were only able to fit the gamma-ray flux of PSR J2021+4026; therefore, we fixed the spectral parameters in each state to the values from the global model. We obtained estimates of the relative variations at the jumps starting from the mean values and the standard deviations in intervals $\mathrm{A}, \mathrm{B}, \mathrm{C}$ and $\mathrm{D}$. In particular, we measured $\Delta F_{\gamma} / F_{\gamma}=-16 \pm 6 \%$ in 2011, $\Delta F_{\gamma} / F_{\gamma}=-13 \pm 5 \%$ in 2018 .

We also studied the timing variability by performing an $H$-test [12] on time intervals of 60 days. This way we could relate flux jumps to the corresponding changes in frequency, $f$, and spin-down rate, $\dot{f}$ (Figure 3 ). We obtained $\Delta \dot{f} / \dot{f}=5 \pm 3 \%$ at the 2011 jump, $\Delta \dot{f} / \dot{f}=5 \pm 2 \%$ at the 2018 jump.

\section{Discussion and conclusions}

According to a previously proposed model [6], the changes in spin-down rate and luminosity of the pulsar could be produced by a shift in the magnetic inclination angle, $\alpha$. Such shift may be the consequence of a glitch induced by a starquake. 

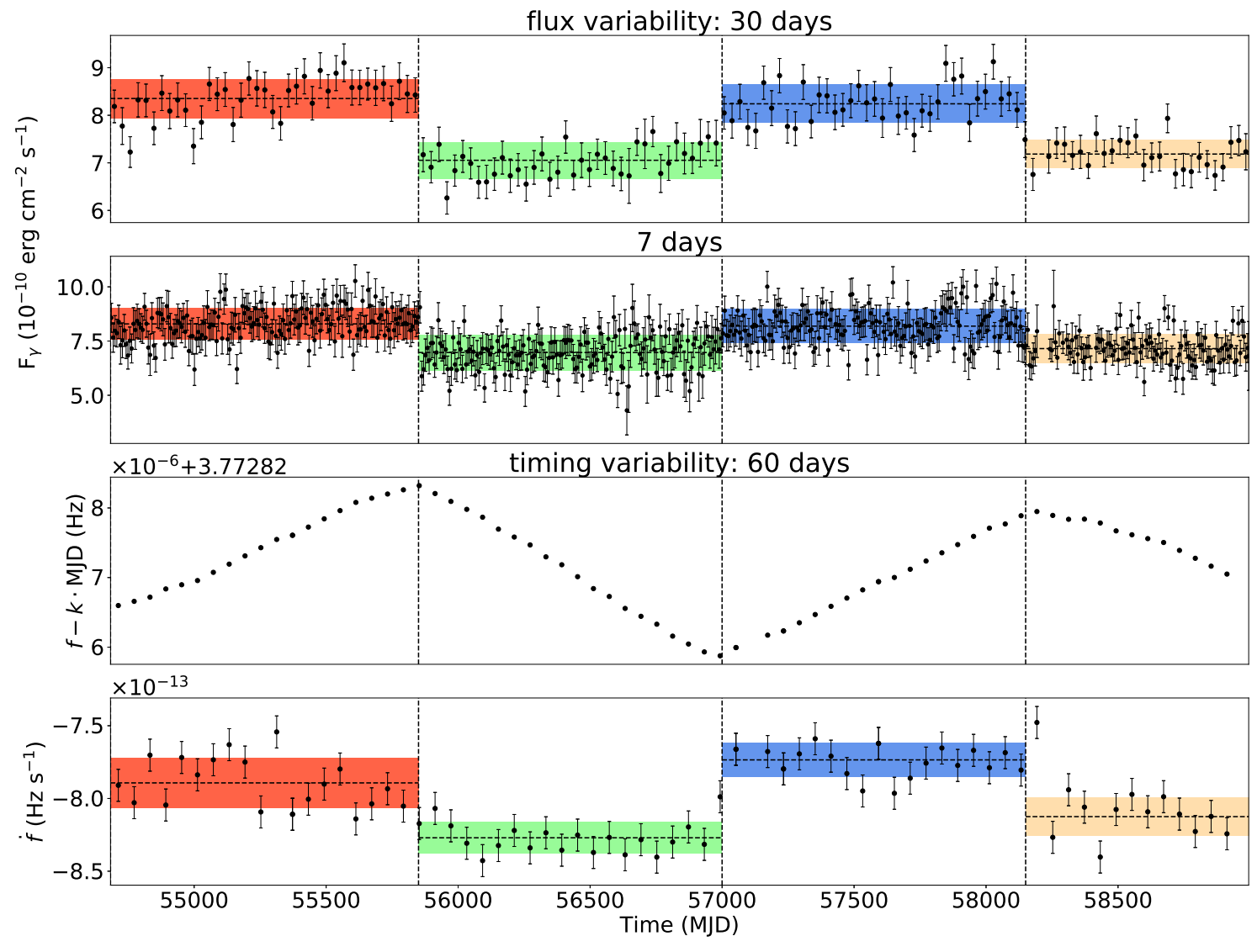

Figure 3: Fitted energy flux and optimal timing parameters of PSR J2021+4026 in the time range from August 5, 2008 to May 26, 2020. Rather than the frequency, $f-k \cdot$ MJD is reported, where $k=6.844 \times 10^{-8}$ $\mathrm{Hz} \mathrm{day}^{-1}$ is a global spin-down rate obtained from a $\chi^{2}$ fit to the $f$ data. Horizontal dashed lines and shaded bands represent the mean values and the $1 \sigma$ confidence bands in the time intervals A (red), B (green), C (blue) and D (orange). Vertical dashed lines indicate the boundaries of the time intervals.

A numerical solution of the equations for magnetohydrodynamics in a force-free magnetosphere [13] gives a formula for the $\gamma$-ray spin-down luminosity:

$$
L_{\mathrm{sd}} \propto f^{4}\left(1+\sin ^{2} \alpha\right)
$$

By setting $L_{\mathrm{sd}}=\dot{E}_{\text {rot }}$ we get

$$
\Delta \dot{f} / \dot{f}=\sin 2 \alpha \Delta \alpha\left(1+\sin ^{2} \alpha\right)^{-1}
$$

If we assume a pre-jump inclination angle $\alpha=63^{\circ}$, obtained from a model of the magnetosphere [6], and the spin-down variation we measured, $\Delta \dot{f} / \dot{f}=5 \pm 2 \%$, we get $\Delta \alpha=6 \pm 2^{\circ}$ and $\Delta L_{\mathrm{sd}} / L_{\mathrm{sd}}=$ $5.0 \pm 1.7 \%$. The latter value is not consistent with the measured relative flux variations, indicating that this simple model is not sufficient to describe the behavior of PSR J2021+4026. Further information may be obtained from a detailed study of the pulse profile, which could strengthen or disprove this hypothesis. 
More sophisticated models [14] are able to produce detailed magnetospheric structures; however, they are valid only in the stationary case. For this reason, we must search hints about the dynamics of the variations by fitting these models to the observed $\gamma$-ray emission in the different states. In this context, the variability analysis of $\gamma$-ray pulsars has a major importance.

Due to its uniqueness, PSR J2021+4026 plays a key role in pulsar physics. Therefore, we are monitoring and analyzing its $\gamma$-ray flux continually. This investigation may represent the starting point of a fine search for variable $\gamma$-ray pulsars. Hints of flux variability in other $\gamma$-ray pulsars have already appeared in the 4FGL catalog, as the case of PSR J2043+2740 [11]. For this reason, we believe that this approach will produce significant results and will lead to a deeper knowledge of the physics of pulsar magnetospheres.

\section{Acknowledgments}

The Fermi-LAT Collaboration acknowledges support for LAT development, operation and data analysis from NASA and DOE (United States), CEA/Irfu and IN2P3/CNRS (France), ASI and INFN (Italy), MEXT, KEK, and JAXA (Japan), and the K.A. Wallenberg Foundation, the Swedish Research Council and the National Space Board (Sweden). Science analysis support in the operations phase from INAF (Italy) and CNES (France) is also gratefully acknowledged. This work performed in part under DOE Contract DE-AC02-76SF00515.

\section{References}

[1] W.B. Atwood et al., The Large Area Telescope on the Fermi Gamma-Ray Space Telescope Mission, ApJ 697 (2009) 1071 [0902 . 1089].

[2] A.A. Abdo et al., Detection of 16 Gamma-Ray Pulsars Through Blind Frequency Searches Using the Fermi LAT, Science 325 (2009) 840 [1009. 0748].

[3] M.C. Weisskopf et al., The Identification of the X-Ray Counterpart to PSR J2021+4026, ApJ 743 (2011) 74 [1108 . 3987].

[4] L.C.C. Lin et al., Discovery of X-Ray Pulsation from the Geminga-like Pulsar PSR J2021+4026, ApJL 770 (2013) L9 [1305 . 0998].

[5] A. Allafort et al., PSR J2021+4026 in the Gamma Cygni Region: The First Variable $\gamma$-Ray Pulsar Seen by the Fermi LAT, ApJL 777 (2013) L2 [1308 . 0358].

[6] C.W. Ng, J. Takata and K.S. Cheng, Observation and Simulation of the Variable Gamma-ray Emission from PSR J2021+4026, ApJ 825 (2016) 18 [1605 . 08968].

[7] J. Zhao et al., Mode Change of a Gamma-Ray Pulsar, PSR J2021+4026, ApJ 842 (2017) 53 [1706.00236].

[8] J. Takata et al., Repeated State Change of Variable Gamma-Ray Pulsar PSR J2021+4026, ApJ 890 (2020) 16 [2001.00289]. 
[9] A.M. Archibald, I.H. Stairs, S.M. Ransom, V.M. Kaspi, V.I. Kondratiev, D.R. Lorimer et al., A Radio Pulsar/X-ray Binary Link, Science 324 (2009) 1411 [0905 . 3397].

[10] B.W. Stappers et al., A State Change in the Missing Link Binary Pulsar System PSR J1023+0038, ApJ 790 (2014) 39 [1311 . 7506].

[11] S. Abdollahi et al., Fermi Large Area Telescope Fourth Source Catalog, ApJS 247 (2020) 33 [1902.10045].

[12] O.C. de Jager and I. Büsching, The H-test probability distribution revisited: improved sensitivity, A\&A 517 (2010) L9 [1005 . 4867].

[13] A. Spitkovsky, Time-dependent Force-free Pulsar Magnetospheres: Axisymmetric and Oblique Rotators, ApJL 648 (2006) L51 [astro-ph/0603147].

[14] C. Kalapotharakos et al., Three-dimensional Kinetic Pulsar Magnetosphere Models: Connecting to Gamma-Ray Observations, ApJ 857 (2018) 44 [1710.03170]. 